American Journal of Pharmacology and Toxicology 2 (4): 148-158, 2007

ISSN 1557-4962

(C) 2007 Science Publications

\title{
Effects of D- and L-Glucose and Mannitol on Retinal Capillary Cells: Inhibition by Nanomolar Aminoguanidine
}

\author{
${ }^{1}$ Yongxin $\mathrm{Yu},{ }^{2} \mathrm{Wei} \mathrm{Li},{ }^{2}$ Barbara Wojciechowski, ${ }^{1,2}$ Alicia J. Jenkins and ${ }^{1,2}$ Timothy J. Lyons \\ ${ }^{1}$ Section of Endocrinology and Diabetes, University of Oklahoma Health Sciences Center \\ WP1345, Oklahoma City, OK 73104 \\ ${ }^{2}$ Division of Endocrinology, Diabetes and Medical Genetics, \\ Medical University of South Carolina, Charleston, SC 29425, USA
}

\begin{abstract}
Hyperglycemia may contribute directly to pericyte loss and capillary leakage in early diabetic retinopathy. To elucidate relative contributions of glycation, glycoxidation, sugar autoxidation, osmotic stress and metabolic effects in glucose-mediated capillary damage, we tested the effects of D-glucose, L-glucose, mannitol and the potentially protective effects of aminoguanidine on cultured bovine retinal capillary pericytes and endothelial cells. Media (containing $5 \mathrm{mM}$ D-glucose) were supplemented to increase the concentration of each sugar by 5, 10, or $20 \mathrm{mM}$. Subconfluent pericytes and endothelial cells were exposed to the supplemented media in the presence or absence of aminoguanidine $(1 \mathrm{nM}-100 \mu \mathrm{M})$ for three days. Cell counts, viability and protein were determined. For both cell types, all three sugars produced concentration-dependent decreases in cell counts and protein content $(p<0.001)$, with D-glucose being the most toxic $(p<0.001)$. The effects of L-glucose and mannitol were similar, suggesting an osmotic contribution to the observed toxicity. Very low concentrations of aminoguanidine, in the range $0.1-1 \mu \mathrm{M}$, abolished the toxicity of all three sugars towards both cell types. The data suggest that D-glucose-mediated toxicity has both intra- and extracellular components and that both can be blocked by aminoguanidine. The efficacy of aminoguanidine at nanomolar concentrations suggests an action through scavenging reactive carbonyls (whether generated by oxidative or metabolic processes) and/or by enzyme inhibition. In addition, aminoguanidine may protect against the consequences of osmotic stress.
\end{abstract}

Key words: Diabetes, retinopathy, endothelial cells, pericytes, toxicity

\section{INTRODUCTION}

Diabetic retinopathy remains the commonest cause of blindness in the working age population. Its pathogenesis is poorly understood $\mathrm{d}^{[1,2]}$, but risk factors include poor glycemic control, long duration of diabetes, hypertension, genetic factors and perhaps hyperlipidemia ${ }^{[1-8]}$. Retinal capillaries are the site of initial damage: compared with other capillaries, their pericytes are unusually numerous, controlling both blood flow and endothelial cell growth, while their endothelial cells possess tight junctions which constitute the inner blood retinal barrier ${ }^{[9,10]}$. Early retinopathy is characterized by pericyte loss and endothelial injury reflected by leakage of the inner blood retinal barrier ${ }^{[10]}$.

Intensive management of diabetes slows the progression of retinopathy ${ }^{[7]}$, consistent with the notion that hyperglycemia contributes directly or indirectly to capillary injury. Potential glycemia-dependent mechanisms include (a) direct "metabolic" (i.e., intracellular) effects of glucose, (b) osmotic effects and (c) effects (either intra- or extracellular) mediated through non-enzymatic glycation, glycoxidation and lipoxidation. These mechanisms may interact. For example, damaging reactive carbonyl-containing intermediates may be formed either by metabolic pathways (e.g., methylglyoxal via the triose phosphate pathway ${ }^{[11]}$ ) or by glycoxidation/lipoxidation reactions (reviewed in 12). Reactive carbonyl groups may subsequently modify macromolecules including proteins $^{[12]}$, phospholipids ${ }^{[13]}$ and nucleotides ${ }^{[14]}$, altering their function and potentially mediating cell injury and death.

Aminoguanidine inhibits the development and progression ${ }^{[15-19]}$ of diabetic retinopathy in both small and large animal models and prevents the increase in permeability of cultured retinal endothelial cells

Corresponding Author: Timothy J. Lyons, Section of Endocrinology and Diabetes,

University of Oklahoma Health Sciences Center, Oklahoma City, OK 73104 
exposed to high glucose $\mathrm{e}^{[20]}$. Also, in diabetic animals it inhibits the development of atherosclerosis ${ }^{[21]}$, nephropathy ${ }^{[22]}$ and neuropathy ${ }^{[23]}$. Its effects are thought to be mediated (a) by inhibition of advanced glycation reactions and/or antioxidant effects ${ }^{[24,25]}$ and/or (b) by inhibition of Nitric Oxide Synthase $(\mathrm{NOS})^{[26,27]}$ or other enzymes such as polyamine oxidase $^{[28]}$, an enzyme involved in the interconversion pathway of polyamines (compounds required for cell proliferation and differentiation). In relation to advanced glycation, aminoguanidine scavenges the reactive carbonyl intermediates described above. It is particularly effective at scavenging dicarbonyl compounds such as glyoxal and 3-deoxyglucosone formed during glycoxidation/lipoxidation and methylglyoxal formed by the triose phosphate pathway $^{[11]}$ (Fig. 1). In relation to NOS inhibition, concentrations of 6-10 $\mu \mathrm{M}$ aminoguanidine are required for $50 \%$ inhibition of inducible NOS (iNOS) in intact cells (insulinoma, neuronal cells), while $100 \mu \mathrm{M}$ is needed for $50 \%$ inhibition of constitutive NOS (in neuronal cells) ${ }^{[27]}$. In retinal capillaries, iNOS has been demonstrated in both endothelial cells and pericytes, but constitutive NOS is found in endothelial cells only ${ }^{[29]}$. Aminoguanidine at high micromolar or millimolar concentrations has been shown to prevent apoptosis: this effect is attributed both to blocking oxidant-induced apoptosis ${ }^{[25]}$ and to iNOS inhibition ${ }^{[30]}$.

We aimed to elucidate the means by which hyperglycemia may injure retinal capillary pericytes and endothelial cells. Cells grown in $5 \mathrm{mM}$ D-glucose were exposed to additional D-glucose, L-glucose and mannitol (by adding $0,5,10$ and $20 \mathrm{mM}$ ), producing total sugar concentrations commonly attained in the diabetic state $(5,10,15$ and $25 \mathrm{mM})$. D-glucose may

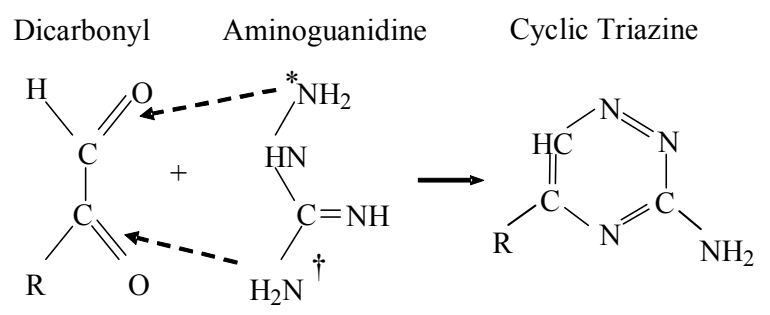

Fig. 1: Action of aminoguanidine in scavenging dicarbonyl-containing compounds. Such compounds are formed as products of free radical oxidation of both carbohydrates and lipids. Initially one amino group (denoted *) binds to either carbonyl, subsequently the second amino group (denoted $=$ ) binds to the second carbonyl group, forming a cyclic triazine have toxic effects mediated through all the actions listed above. Its stereo-isomer, L-glucose, is not metabolized by cells, but can participate in extracellular glycation and glycoxidation reactions and has osmotic effects. L-glucose can enter at least some cell types (erythrocytes) perhaps by mass action ${ }^{[31]}$ and may therefore lead to intracellular glycation. Mannitol does not partake in glycation reactions and is used as an osmotic control.

We also assessed the ability of aminoguandine to inhibit D-glucose-mediated toxicity towards retinal capillary cells. To maintain patho-physiologic and pharmacologic relevance, we first used low micromolar concentrations of aminoguanidine (range: 1-100 $\mu \mathrm{M}$ ), i.e., levels at or below those in plasma and tissues of aminoguanidine-treated animals ${ }^{[16]}$ and humans ${ }^{[32]}$. The abolition of toxic effects at even the lowest of these concentrations led to a second series of experiments, in which levels of aminoguanidine one thousand-fold lower (nanomolar concentrations) were used to define dose-response.

\section{MATERIALS AND METHODS}

Reagents: Aminoguanidine was a gift from Alteon Inc., Northvale, NJ. All other reagents were obtained from Sigma Chemical Company, St. Louis, MO, unless otherwise specified.

Culture of bovine retinal capillary endothelial cells and pericytes: Bovine globes from freshly-killed animals were transported promptly (within $4 \mathrm{~h}$ of death), on ice, to our laboratory. Retinal capillary pericytes and endothelial cells were isolated and cultured as previously described ${ }^{[33]}$ using a modification of the method of King et al ${ }^{[34]}$. Briefly, pericytes were cultured in Dulbecco's Modified Eagle's Medium (DMEM) (Whittaker, Walkersville, MD) supplemented with $15 \%$ fetal calf serum, $36 \mathrm{mM} \mathrm{NaHCO}_{3}$, fungizone, streptomycin, kanamycin and benzyl penicillin. The retinal microvessels were plated onto plain plastic tissue culture flasks. Cell purity was assessed morphologically and confirmed by positive staining for actin (Molecular Probes, Eugene, OR) (>95\%) and 3G5-defined ganglioside antigen ${ }^{[35]}(>80 \%)$ and absence of staining for factor VIII antigen ( $>95 \%)$.

Endothelial cells were cultured in DMEM supplemented with $10 \%$ human plasma, $90 \mathrm{mg} \mathrm{L}^{-1}$ heparin, $200 \mu \mathrm{g} \mathrm{L}^{-1}$ insulin, $60 \mathrm{mg} \mathrm{L}^{-1}$ endothelial growth supplement (Chemicon, Temecula, CA) and antimicrobials as above. Microvessels were plated onto gelatinized tissue culture dishes. Cell purity was assessed morphologically and confirmed by positive 
staining for factor VIII antigen (>80\%) (Atlantic Antibodies, Stillwater, MN).

Experiments comparing different sugars: Eight experiments were performed with pericytes and nine with endothelial cells. Within each experiment, cells were incubated for two and three days in growth media containing $5 \mathrm{mM}$ D-glucose supplemented with Dglucose, L-glucose, or mannitol to produce final total sugar concentrations of $5,10,15$, or $25 \mathrm{mM}$. D-glucose, L-glucose and mannitol stocks (400 mM) were prepared as fresh solutions, appropriate dilutions were made and media were supplemented with the sugars immediately before addition to cells.

In each experiment, cells (Passage 2-6) were plated into five (12-well) plates (Costar, Cambridge, MA) and allowed to seed and reach sub-confluence (over 23 days) before changing to experimental growth media. Day 0 denotes the time at which cells were changed to experimental media. One plate was used at Day 0 for baseline determinations (six wells for cell counts, six for cell protein); the remaining plates were used for the study conditions (two at each time point, Day 2 and Day 3). After incubation, media were aspirated and cell counts, cell viability (using Trypan Blue) and protein were determined. There were two wells for each of the twelve conditons (three sugars $x$ four sugar concentrations). Of each pair of wells, one was used for cell counting, the other for protein determination.

Experiments using aminoguanidine: In these experiments, in order to study multiple aminoguanidine concentrations, the Day 2 time point and intermediate sugar concentrations were omitted. Two series of experiments were conducted using different ranges of aminoguanidine with 5 and $25 \mathrm{mM}$ D-glucose. The first series employed aminoguanidine at micromolar concentrations $\quad\left(\begin{array}{lllll}1-100 & \mu \mathrm{M}\end{array}\right)$. Because $1 \quad \mu \mathrm{M}$ aminoguanidine was as effective as $100 \mu \mathrm{M}$, the second series was performed to seek dose-response information at lower, nanomolar, concentrations $(0.001-1 \mu \mathrm{M})$. A small third series of experiments was also performed to assess the efficacy of aminoguanidine in the presence of $25 \mathrm{mM}$ L-glucose and mannitol.

Control and experimental media were prepared as follows. Aminoguanidine (7 parts of stock solution ( 0 , $0.1,1.0$, or $10 \mathrm{mM}$ for first series; lower concentrations for second series) and D-glucose (5.1 parts of 0 or $2.77 \mathrm{M}(50 \% \mathrm{D}$-glucose)) were first mixed together, then $120 \mu \mathrm{L}$ of the mixture were added promptly to $6.88 \mathrm{~mL}$ fresh growth media (added volume $<2 \%$ of total). Media were then added to cells. At the $10 \mu \mathrm{M}$ aminoguanidine condition (first series only), a variant protocol was tried: Glucose was added to the media first, followed 15 min later by aminoguanidine and media were then placed on cells. This variant did not alter any outcome measure and results are not presented.

In the first series (higher aminoguanidine concentrations), 18 experiments were performed with pericytes and 12 with endothelial cells, while in the second (lower concentrations), four experiments were performed with each cell type. In both series, cells were first allowed to reach subconfluence in 12-well plates as described above. Media were then changed to experimental conditions (Day 0) and cells were incubated for a further three days in fresh growth media in the presence of normal or high D-glucose $(5,25 \mathrm{mM})$ with and without aminoguanidine at the following concentrations: first series: $0,1,10,100 \mu \mathrm{M}$, second series: $0,0.001,0.01,0.1$ and $1 \mu \mathrm{ML}$. In the third series, effects of aminoguanidine $(0,1,10,100 \mu \mathrm{M})$ were assessed in the presence of $25 \mathrm{mM}$ sugar achieved by additional L-glucose or mannitol.

Cell counting, cell protein determination: Cells from individual wells were trypsinized, then resuspended in $100 \mu \mathrm{L}$ of growth medium to which $200 \mu \mathrm{L} 1 \%$ Trypan Blue solution was added. Using a hemocytometer, total and live cells in four $0.1 \mu \mathrm{L}$ volumes were counted as described $^{[35]}$. The total of these four cell counts was multiplied by 750 to estimate cells per well. All cell counts were performed by the same observer, who was unaware of sample identity. Cell protein was determined by the method of Lowry as modified by Markwell et al. ${ }^{[36]}$. Statistical analysis was performed using Statistica for Windows, version 5.1 (Statsoft, Inc. Tulsa, OK).

Experiments with different sugars: Multivariate Analysis of Variance (MANOVA) was used to assess differences between the 48 treatment groups (four sugar concentrations $\mathrm{x}$ three types of sugar $\mathrm{x}$ two cell types $\mathrm{x}$ two days of observation). For this purpose, responses (cell counts, cell viability (live cell counts) and cell protein) on Day 2 or Day 3 were expressed as a percentage of those observed on Day 0 (because two study days were involved). Based on the results of MANOVA, appropriate univariate Analysis of Variance (ANOVA) was performed for Day 3 only. For ANOVA, response variables were calculated as percentage of the control condition (5 $\mathrm{mM}$ D-Glucose) on Day 3. Tukey's Honest Significant Difference (HSD) test was used for post hoc comparisons. For illustrative purposes, data in the figures show only Day 3 cell counts $(m e a n \pm S E M)$ : Absolute values for 
both Day 3 and Day 0 are provided in the Legends. To study dose response to sugar concentration, the relationship between toxicity, defined as percent reduction (100-response) and the natural log of sugar concentration was analyzed using linear regression with indicator variables. Intra-experiment coefficients of variation for cell counts, live cell counts and protein were determined at 5.3, 5.2 and $4.3 \%$ respectively for pericytes and $6.1,6.8$ and $3.7 \%$ respectively for endothelial cells ${ }^{[23]}$.

Experiments with aminoguanidine: Univariate ANOVA using response variables calculated as percentage of baseline (Day 3, 5mM D-Glucose, no aminoguanidine) were performed for each response and cell type. For illustrative purposes in the figures, only Day 3 cell counts are shown as mean \pm SEM, as above.

\section{RESULTS}

\section{Experiments with different sugars:}

Global MANOVA: The analysis encompassing all data showed highly significant outcome differences $(p<0.001)$ according to concentration of sugar, type of sugar, cell type and day of observation. There were strong overall correlations between the three outcome variables (total and live cell counts, cell protein) $(p<0.001)$. Since effects on Day 3 were consistent with but more severe than those on Day 2, only data for Day 3 are presented in figures.

Analysis of variance at day 3: Significant differences in responses between the two cell types were observed at Day 3 (ANOVA, Table 1). Pericytes (Table 1) and endothelial cells (Table 1) were therefore analyzed separately and for each, significant differences were seen according to both concentration and type of sugar. There were no interactions between these variables (i.e., they act independently), so each was analyzed separately.

Effect of sugar concentration: Increased sugar concentration (independent of type of sugar) led to significantly decreased cell counts, viability and protein for both cell types. The data are shown in detail in Table 2 and are shown in Fig. 2. The dose-toxicity response for all three sugars combined (expressed as percent reduction in cell counts) was log-linear (Fig. 3) $(\mathrm{r}=0.415, \mathrm{p}<0.001)$. Similar log-linear responses were observed for reductions in live cell counts and cell protein (data not shown).
Table 1: Results of analysis of Variance at Day 3 ( $p$ values). No significant interactions were observed between sugar concentration, sugar type, or cell type

\begin{tabular}{llll}
\hline A: All data & Cell counts & Live cell counts & Cell protein \\
\hline Cell Type & $<0.001$ & $<0.001$ & 0.41 \\
Concentration of sugar & $<0.001$ & $<0.001$ & $<0.001$ \\
Type of sugar & $<0.001$ & $<0.01$ & $<0.001$ \\
B: Pericytes & & & \\
Concentration of sugar & $<0.001$ & $<0.001$ & $<0.001$ \\
Type of sugar & $<0.005$ & 0.055 & 0.01 \\
C: Endothelial cells & & & \\
Concentration of sugar & $<0.001$ & $<0.001$ & $<0.001$ \\
Type of sugar & $<0.02$ & 0.19 & $<0.01$ \\
\hline
\end{tabular}

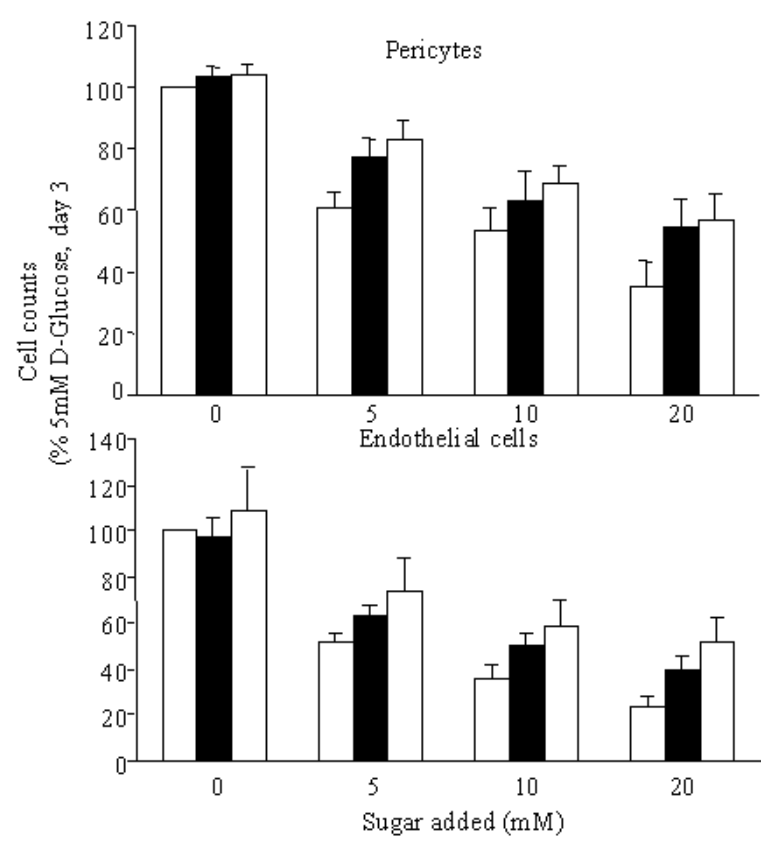

Fig. 2: Cell counts for retinal capillary pericytes (top panel, $\mathrm{n}=8$ ) and endothelial cells (bottom panel, $\mathrm{n}=9$ ) following three days' exposure to media containing $5 \mathrm{mM}$ D-glucose and additional Dglucose (open bars), L-glucose (black bars) and mannitol (shaded bars). Data are expressed as percent of counts at Day 3 (mean \pm SEM) for the D-glucose control condition (shown as 100\%, no error bars). Mean Day 3 counts in the control condition for pericytes were $169.2 \pm 10.9 \times 10^{3}$ cells/well and for endothelial cells, $125.7 \pm 31.8 \times 10^{3}$ cells/well. Baseline (Day 0) counts for pericytes were $144.9 \pm 19.0 \times 10^{3}$ cells/well and for endothelial cells, $72.3 \pm 14.9 \times 10^{3}$ cells/well. See Tables and text for statistical analysis

Effect of type of sugar: The toxicity of D-Glucose was higher overall than the other two sugars $(\mathrm{p}<0.001$, 
Am. J. Pharm. \& Toxic. 2 (4): 148-158, 2007

Table 2: Tukey's pairwise comparisons, concentration of sugar, day 3

\begin{tabular}{lllll}
\hline Added [Sugar], mM & 0 & 5 & 10 & 20 \\
\hline Pericytes & & & & \\
0 & - & C, L, P & C, L, P & C, L, P \\
5 & C, L, P & - & ns & C, L, P \\
10 & C, L, P & Ns & - & P \\
20 & C, L, P & C, L, P & P & - \\
Endothelial cells & & & & \\
0 & - & C, L,P & C, L, P & C, L, P \\
5 & C, L, P & - & ns* & C, L, P \\
10 & C, L, P & ns* & - & P \\
20 & C, L, P & C, L, P & P & - \\
\hline
\end{tabular}

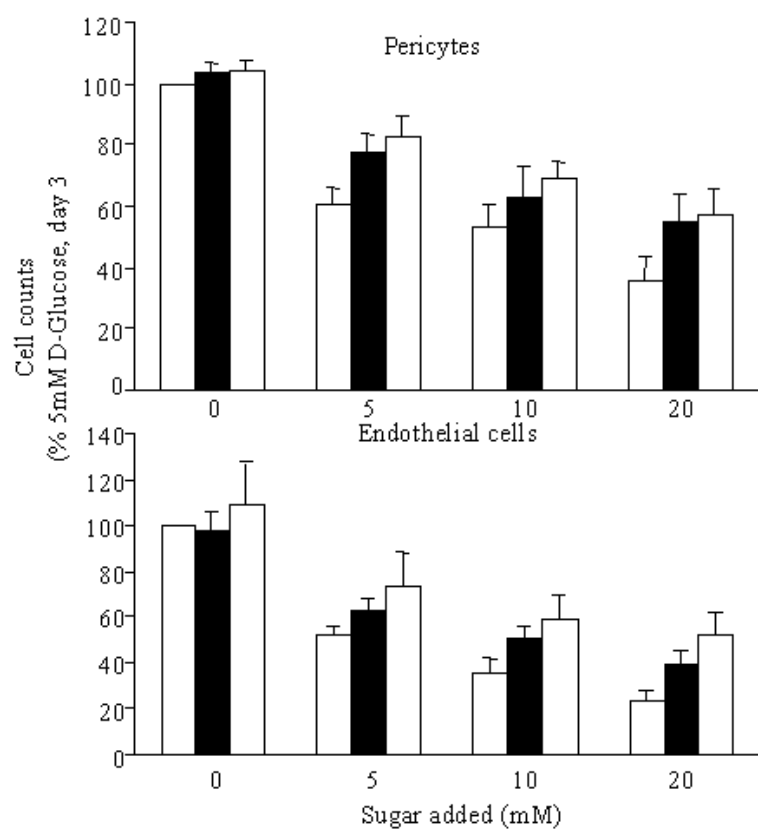

Fig. 2: Cell counts for retinal capillary pericytes (top panel, $\mathrm{n}=8$ ) and endothelial cells (bottom panel, $\mathrm{n}=9$ ) following three days' exposure to media containing $5 \mathrm{mM}$ D-glucose and additional Dglucose (open bars), L-glucose (black bars) and mannitol (shaded bars). Data are expressed as percent of counts at Day 3 (mean \pm SEM) for the D-glucose control condition (shown as $100 \%$, no error bars). Mean Day 3 counts in the control condition for pericytes were $169.2 \pm 10.9 \times 10^{3}$ cells/well and for endothelial cells, $125.7 \pm 31.8 \times 10^{3}$ cells/well. Baseline (Day 0) counts for pericytes were $144.9 \pm 19.0 \times 10^{3}$ cells/well and for endothelial cells, $72.3 \pm 14.9 \times 10^{3}$ cells/well. See Tables and text for statistical analysis

ANOVA): This is illustrated for cell counts in Fig. 2 and 3. Significant differences for the individual responses are shown in Table 3: D-glucose produced
Table 3: Tukey's pairwise comparisons, type of sugar

\begin{tabular}{llll}
\hline & D-glucose & L-glucose & Mannitol \\
\hline Pericytes & - & & \\
D-glucose & - & $\mathrm{C}^{*}$ & $\mathrm{C}, \mathrm{P}^{* *}$ \\
L-glucose & $\mathrm{C}^{*}$ & - & $\mathrm{ns}$ \\
Mannitol & $\mathrm{C}, \mathrm{P}^{* *}$ & $\mathrm{~ns}$ & - \\
Endothelial cells & & & \\
D-glucose & - & $\mathrm{P}$ & $\mathrm{C}, \mathrm{P}$ \\
L-glucose & $\mathrm{P}$ & - & $\mathrm{ns}$ \\
Mannitol & $\mathrm{C}, \mathrm{P}$ & $\mathrm{ns}$ & - \\
\hline
\end{tabular}

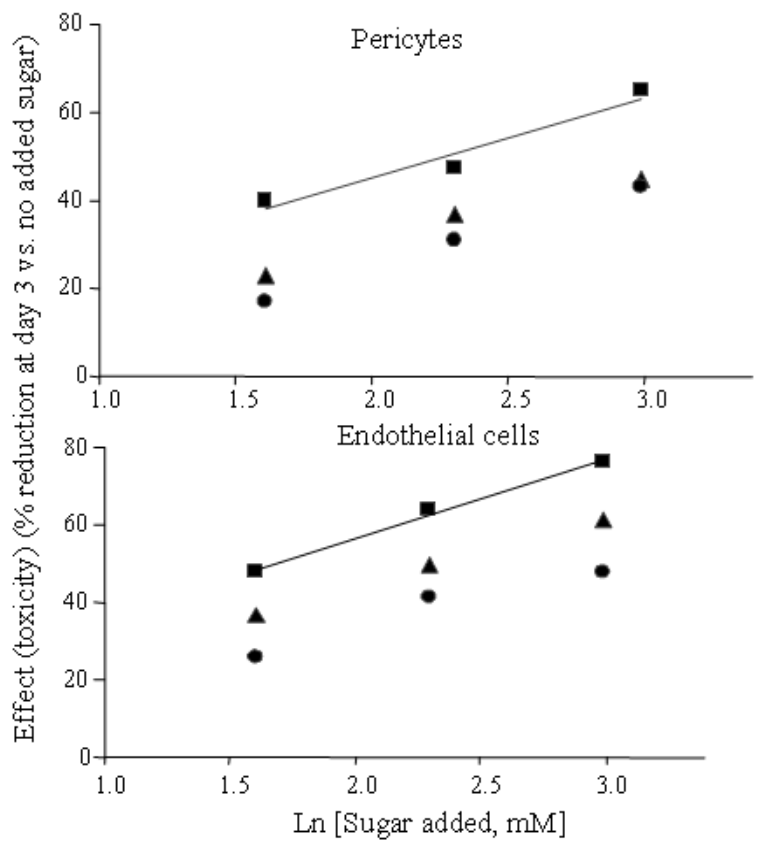

Fig. 3: Dose response curves showing log-linear toxic effects of added D-glucose ( $(\boldsymbol{\square})$, L-glucose $(\boldsymbol{\Delta})$ and mannitol $(\diamond)$ towards pericytes and endothelial cells. Data are derived from those shown in Fig. 1, error bars are omitted for clarity. D-glucose was significantly more toxic than the other two sugars $(\mathrm{p}<0.001$, ANOVA)

significantly greater decreases in cell counts and protein than mannitol and for some outcomes, than L-glucose. Generally the responses to L-glucose and mannitol were similar.

Table 2 shows where differences $(\mathrm{p}<0.05)$ were found according to sugar concentration. Parameters are denoted as follows: $\mathrm{C}=$ Cell counts, $\mathrm{L}=$ Live cell counts, $\mathrm{p}=$ Cell protein, $\mathrm{ns}=$ No significance. Shaded areas: Redundant information included for clarity. Example: For pericytes, cell protein was significantly different after three days' exposure to $20 \mathrm{mM}$ v $10 \mathrm{mM}$ added sugar, but no significant differences were observed for cell counts or live cell counts. *: Trends: for $\mathrm{C}, \mathrm{p}=0.067$, for $\mathrm{L}, \mathrm{p}=0.054$. 


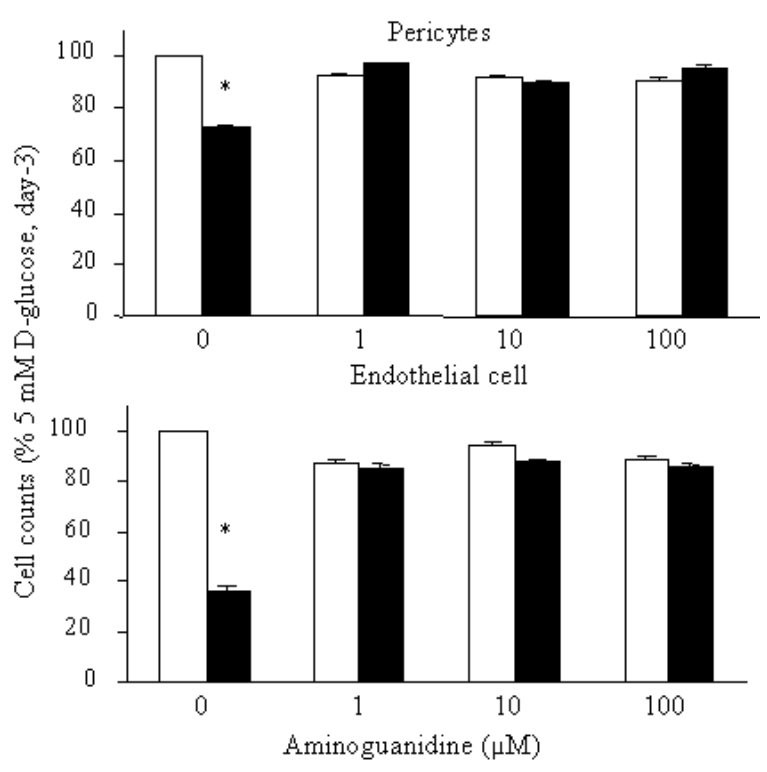

Fig. 4: Cell counts for pericytes $(\mathrm{n}=18)$ and endothelial cells $(\mathrm{n}=12)$ after 3 days' exposure to $5 \mathrm{mM}$ D-glucose (white bars) and $25 \mathrm{mM} \mathrm{D-}$ glucose (black bars) in the absence and presence of aminoguanidine, 1-100 $\mu \mathrm{M}$. At each concentration, aminoguanidine abolished the toxic effects of $25 \mathrm{mM}$ D-glucose towards both cell types. Cell counts (mean \pm SEM) are expressed as $\%$ of counts in the control condition (5 $\mathrm{mM}$ D-glucose, no aminoguanidine) at Day 3. Mean Day 3 counts in the control condition for pericytes were $171.0 \pm 19.1 \times 10^{3}$ cells/well and for endothelial cells, $128.6 \pm 14.2 \times 10^{3}$ cells/well. Baseline (Day $0)$ counts for pericytes were $109.5 \pm 14.0 \times 10^{3}$ cells/well and for endothelial cells, $85.1 \pm 9.9 \times 10^{3}$ cells/well. Statistical analysis: *: Significantly different from all other conditions $(p<0.05$, Tukey's Honest Significant Difference)

Table 3 shows where differences $(p<0.05)$ were found according to type of sugar at Day 3. Parameters and abbreviations are as denoted in Table 2. Example: total pericyte counts were significantly different after three days' exposure to D-glucose than to L-glucose, but no significant differences were observed for live cell counts or cell protein between these two sugars. Trends: * for live cell counts, $p=0.11, * *$ for live cell counts, $p=0.08$.

\section{Experiments with Aminoguanidine}

First series (micromolar concentrations of aminoguanidine, normal and high D-glucose): In this series, all concentrations of aminoguanidine employed $(1,10,100 \mu \mathrm{M})$ abolished the toxicity of $25 \mathrm{mM}$ Dglucose, i.e., $1 \mu \mathrm{M}$ was as effective as $100 \mu \mathrm{M}$. This effect is illustrated for cell counts in Fig. 4. Significant
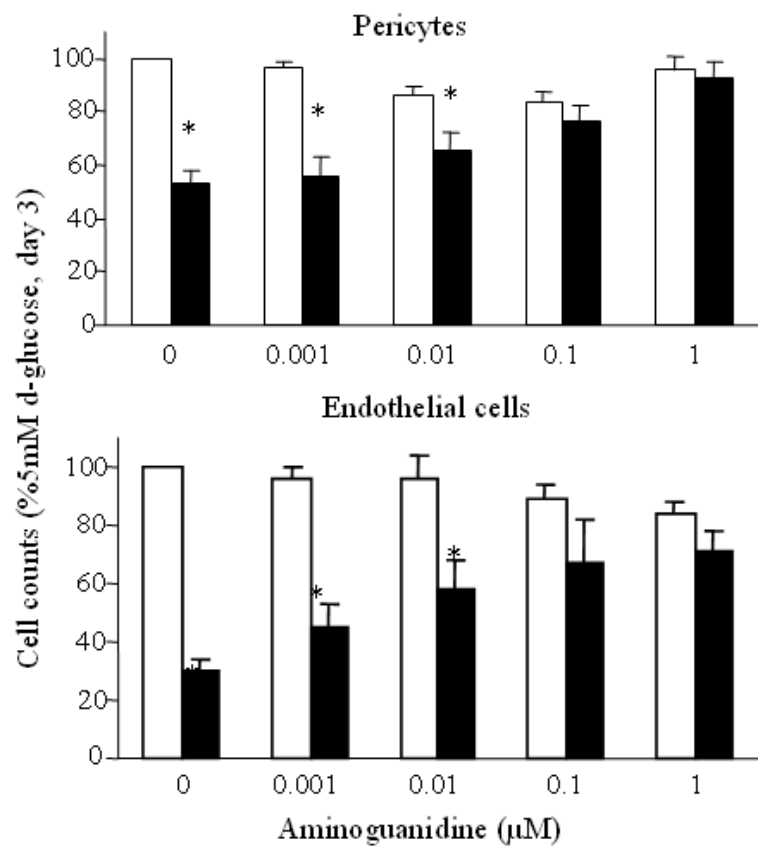

Fig. 5: Cell counts for pericytes $(n=4)$ and endothelial cells $(\mathrm{n}=4)$ after 3 days' exposure to $5 \mathrm{mM}$ (white bars) and $25 \mathrm{mM}$ D-glucose (black bars) in the absence and presence of aminoguanidine, 0.001-1 $\mu \mathrm{M}$. At these concentrations, aminoguanidine mitigated the toxic effects of $25 \mathrm{mM}$ D-glucose for each cell type in a dosedependent fashion. Cell counts (mean \pm SE) are expressed as in Fig. 4. Mean Day 3 counts in the control condition for pericytes were $174.0 \pm 15.4$ cells/well and for endothelial cells, 3 were $108.0 \pm 4.8$ cells/well. Baseline (Day 0) counts for pericytes were $142.9 \pm 14.6 \times 10^{3}$ cells/well and for endothelial cells, $51.3 \pm 7.9 \mathrm{v} 10^{3}$ cells/well. Statistical analysis: * significantly different from corresponding $5 \mathrm{mM}$ conditions $(p<0.05$, Tukey's Honest Significant Difference)

differences were observed only between the high glucose/zero aminoguanidine condition and all others $(\mathrm{p}<0.001$ in each case). The aminoguanidine concentrations were chosen for pharmacologic relevance, but the results led to a second series of experiments to investigate dose reponses at lower concentrations.

Second series (nanomolar concentrations of aminoguanidine, normal and high D-glucose): A dose-dependent protective effect of aminoguanidine at lower, nanomolar concentrations was observed. This effect is illustrated for cell counts in Fig. 5. 
Am. J. Pharm. \& Toxic. 2 (4): 148-158, 2007

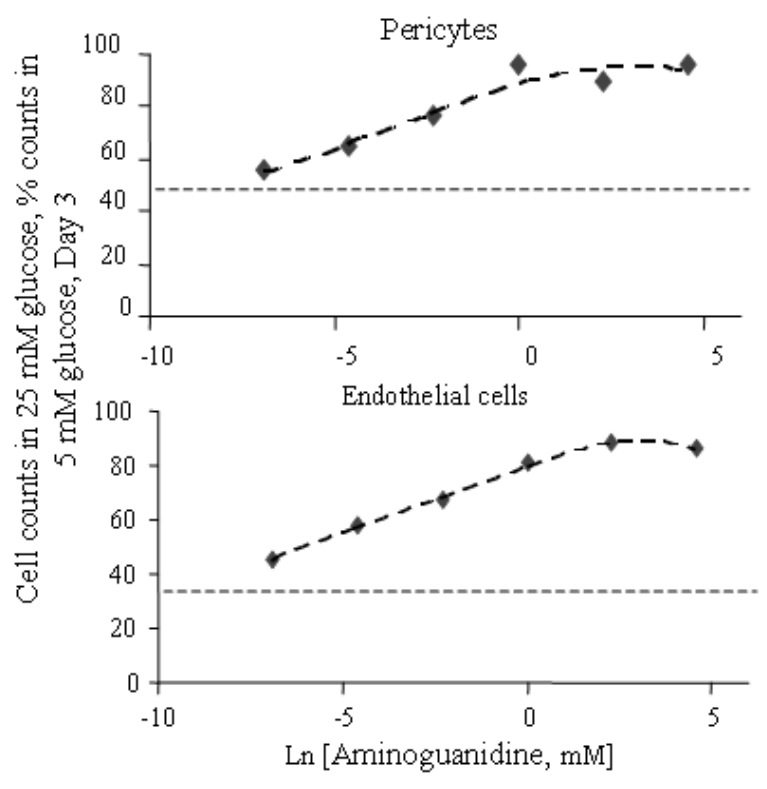

Fig. 6: Dose response curves for aminoguanidine (0.001-100 $\mu \mathrm{M})$ in mitigating toxic effects of $25 \mathrm{mM}$ glucose towards pericytes and endothelial cells. The hatched lines represent cell counts in $25 \mathrm{mM}$ glucose with no aminoguanidine. For both cell types, the relationship is log-linear up to $1 \mu \mathrm{M}$ aminoguanidine. At and above this concentration, toxicity is abolished

Concentrations between 0.01 and $1.0 \mu \mathrm{M}$ were sufficient to abolish significant D-glucose-mediated toxicity.

No toxicity of aminoguanidine towards cells in the presence of $5 \mathrm{mM}$ D-glucose was observed in either series $(p=n s$, ANOVA). Cell count data from both series are combined and summarized in Fig. 6. For each cell type, the dose-response curve is log-linear over the aminoguanidine concentration range $1 \mathrm{nM}$ to $1 \mu \mathrm{M}$, above which it reaches a plateau.

Third series (aminoguanidine with L-glucose, mannitol): A small, third series of experiments was performed to investigate the effects of aminoguanidine in the higher concentration range $(1,10,100 \mu \mathrm{M})$ on the effects of $25 \mathrm{mM}$ sugar attained by supplementing media with L-glucose or mannitol. With both cell types, as with D-glucose, the (lesser) toxicities of these sugars were abolished by $1 \mu \mathrm{M}$ aminoguanidine $(\mathrm{n}=4$, data not shown).

\section{DISCUSSION}

In these experiments, elevated concentrations of all three sugars significantly reduced retinal capillary cell numbers and cell protein, but the reductions were most severe following exposure to elevated D-glucose. The findings are consistent with toxic effects of D-glucose additional to those of the other sugars, presumably dependent upon its entry into cells and subsequent metabolism. L-glucose and mannitol had toxicities similar to one another and less severe than D-glucose.

Since both these sugars exert osmotic effects but only L-glucose glycates, the results imply that extracellular glycation reactions contribute little to the observed sugar toxicity. The extent to which L-glucose enters cultured retinal capillary cells is unknown, but is likely to be small compared with D-glucose. It therefore appears that the toxicity of D-glucose is the result of a combination of extracellular (osmotic or oxidative stresses) and intracellular effects (metabolic, oxidative, or glycation-associated stresses).

Aminoguanidine protected against the toxicity of all three sugars, implying efficacy against both the extra- and intra-cellular components of D-glucose toxicity. Previously Chibber et al. ${ }^{[37]}$ showed that high, pharmacologically irrelevant concentrations of aminoguanidine $\left(1-10 \mathrm{mmol} \mathrm{L}^{-1}\right.$, i.e., many orders of magnitude greater than those we employed) blocked the anti-proliferative effects of high glucose towards retinal capillary pericytes. Our results were surprising in view of the very low effective concentrations of aminoguanidine, particularly if the extracellular component of sugar toxicity is predominantly mediated by osmotic stress. Hypertonic mannitol has been previously shown to induce vascular endothelial cell apoptosis, accompanied by activation of tyrosine and stress kinases, phosphorylation of adhesion contactassociated proteins and elevation of intracellular free calcium $^{[38]}$. The mechanism of aminoguanidine in inhibiting this process is unknown. A protective effect from oxidative stress could also be implicated, since oxidative stress is increased in the presence of elevated glucose $^{[39]}$. Osmotic and oxidative stresses may activate closely related or identical intracellular signaling pathways ${ }^{[40]}$, perhaps providing therapeutic opportunities to reduce the effects of both stresses simultaneously; however, such an action for aminoguandine is entirely speculative.

Overproduction of superoxide by the mitochondrial electron-transport chain is regarded to be the central mechanism mediating hyperglycemida-induced cellular injury in diabetes ${ }^{[41]}$. It has been shown that high glucose $(30 \mathrm{mM})$ increased intracellular reactive oxygen species (ROS) generation in cultiured retinal pericytes, which was completely blocked by the pigment epithelium-derived factor (PEDF ${ }^{[42]}$. PEDF also blocks high glucose or hydrogen peroxide induced peicyte 
apoptosis, at least partially through its antioxidant effects via glutathione peroxidase induction ${ }^{[42]}$. There is evidence that aminoguanidine also has direct antioxidant effects and this could be pertinent to our observations: millimolar concentrations have been shown to inhibit the oxidation of $\mathrm{LDL}^{[43]}$, but at lower concentrations $(10 \mu \mathrm{M})$, aminoguanidine may actually be pro-oxidant ${ }^{[44]}$. Giardino et al. ${ }^{[25]}$ showed that aminoguanidine has antioxidant effects in cultured retinal Müller cells during exposure to hydrogen peroxide-mediated oxidative stress, but the stress employed ( $20 \mu \mathrm{M}$ hydrogen peroxide) was more severe and of a different nature from that used in our experiments. Also, the concentration of aminoguanidine was higher $(100 \mu \mathrm{M})$. Nevertheless it is conceivable that elevated glucose levels and even osmotic stress itself might generate intracellular oxidative stress, triggering apoptosis (as observed in Giardino's work ${ }^{[25]}$ ) and that the lesser stimuli we employed could be blocked by our lower aminoguandine concentrations. Another possibility is that our sugar preparations contained small quantities of carbonyl-containing impurities and that these impurities mediated effects on cells and were scavenged by aminoguanidine. However, in preliminary experiments (data not shown) we exposed our sugars to lipid-free albumin to absorb any such reactive contaminants, then removed the albumin by filtration. This procedure had no effect on sugar toxicity.

The intracellular effects of D-glucose may be mediated by (a) metabolic processes (e.g., Protein Kinase $\mathrm{C}$ activation ${ }^{[45]}$ or increased flux through the triose phosphate ${ }^{[11]}$ or aldose reductase pathways), or (b) intracellular glycation, glycoxidation and lipoxidation reactions ${ }^{12,41]}$. Both the triose phosphate pathway and glycation-associated reactions can generate species containing reactive dicarbonyls: the triose phosphate pathway leads to the formation of methylglyoxal, while autoxidation of glucose and oxidation of lipids may generate glyoxal and other dicarbonyls ${ }^{[12]}$. Furthermore, the non-oxidative decomposition of the initial glycation product fructoselysine results in the formation of a dicarbonylcontaining compound, 3-deoxyglucosone ${ }^{[46]}$. Dicarbonyls such as methylglyoxal and glyoxal have been clearly shown to induce apoptosis in cultured bovine retinal pericytes that involves the mechanims including oxidative stress, NF-kappaB activation ${ }^{[47]}$ and fibronectin modification ${ }^{[48]}$. Aminoguanidine may scavenge these carbonyl-containing species. However, this would require entry of aminoguanidine into cells and to our knowledge, the extent to which aminoguanidine enters cells is unknown and it was not determined in this study.
The mechanims of inhibition of AGEs by aminoguanidine are supported by some animal experiments. Hammes et al. ${ }^{[15,16]}$ observed that aminoguanidine inhibited retinal pathology (acellular capillaries, pericyte dropout, microaneurysms, endothelial proliferation) in diabetic rats and that the drug inhibited the formation of AGEs in retinal microvessels as well as the retinal autofluorescence, an indiactor of AGEs. However, Kern et al. ${ }^{[18]}$ found that the inhibition of retinal lesions by aminoguanidine in diabetic rats occurs without systemic reductions in AGEs, including Hb-AGE (mainly carboxymethyl lysine), retinal pentosidine and tail collagen fluorescence and pentosidine. In a diabetic dog model, which is believed to develop retinopathy morphologically similar to the characteristic of diabetic patients, Kern et al. ${ }^{[19]}$ further showed that aminoguanidine had no significantly beneficial effect on systemic AGEs such as Hb-AGE and pentosidine. However, aminoguanidine tended to inhibit accumulation of retinal imidazolone, although the effect was not statistically significant ${ }^{[18]}$.

Enzyme-inhibiting effects of aminoguanidine could be involved in the effects we observed, although the effective concentrations seem too low to implicate iNOS inhibition in this study ${ }^{[16]}$. Aminoguanidine appeared to inhibit protein nitration in the retina of diabetic dogs and rats, consistent with aminoguanidinemediated inhibition of $\mathrm{iNOS}^{[19,49]}$. A recent study provided evidence that methylglyoxal metabolism and iNOS interact with each other and may be mechanistic in human retinal capillary cell death under hyperglycemic conditions ${ }^{[50]}$. In that study, unlike the bovine conterpart, human retinal pericytes incubated with $30 \mathrm{mM}$ D-glucose for seven days did not undergo apoptosis despite accumulation of methylglyoxal. However, a combined treament of high glucose and an inhibitor of glyoxalase I, the metabolizing enzyme of methylglyoxal, resulted in apoptosis along with a dramatic increase in methylglyoxal; the toxicity was protected by the glyoxalase I overexpression in pericytes. Incubation of pericytes with high concentrations of methylglyoxal also resulted in an increase of apoptosis. When pericytes were incubated with an nitric oxide donor, they observed both decreased glyoxalase I expression and activity and apoptosis and that apoptosis increased further upon coincubation with high glucose. These findings indicate that the level or activity of glyoxalase I (methylglyoxal) is critical for pericyte survival under hyperglycemic conditions and its inactivation or downregulation by nitric oxide (reflecting iNOS function) may contribute to pericyte death. 
The aminoguanidine concentrations which inhibit polyamine oxidase have not been characterized in detail, but those employed in in vitro studies are much higher than in the present study $(500 \mu \mathrm{M})^{[28]}$. Effects on other enzyme systems are possible. For example, aminoguanidine is also a potent inhibitor of Semicarbazide-Sensitive Amine Oxidase (SSAO) both in vitro and in $v i v o^{[51]}$. This enzyme catalyzes the deamination of methylamine and aminoacetone, which leads to the production of cytotoxic formaldehyde and methylglyoxal, respectively. SSAO activity is increased in diabetic patients and positively correlated with the amount of plasma glycated haemoglobin. Again, in cell culture systems, such effects are presumabaly dependent on the entry of aminoguanidine into cells.

A possible mechanism of action less dependent on the entry of aminoguanidine into cells is an effect on cell membrane-dependent signaling processes. In other studies, we have shown that similarly low levels of aminoguanidine (maximal effect at $1 \mu \mathrm{mol} \mathrm{L} \mathrm{L}^{-1}$ ) can inhibit the development of cytotoxicity in Low Density Lipoproteins (LDL) during exposure of LDL to in vitro glycoxidative stress ${ }^{[52]}$. In that work, aminoguanidine was removed from the aqueous phase of the LDL preparation before the lipoprotein was added to cultured retinal capillary cells. It follows that its action in preventing cell death must have involved a prior interaction with D-glucose, oxidants and the lipoprotein surface. An analogous interaction occurred in the present study: aminoguanidine, together with glucose and an oxidant aqueous phase was in contact with a lipid/protein surface, in this case, the cell membrane. The main lipid components of both cell membranes and lipoprotein surfaces are phospholipids and free cholesterol. In cell membranes, the role of membrane lipids in cell signaling processes is increasingly recognized ${ }^{[53]}$ and the ability of glucose and oxidants to modify phospholipids is established ${ }^{[13]}$. It is possible that during exposure of cells to glycoxidative stress, aminoguanidine may inhibit modification of cell membrane components involved in lipid or proteindependent signaling processes. Since these lipid signaling molecules produce substantial signal amplification, a relatively small alteration in input could induce significant changes in cell behavior. Such a mechanism could explain the potent effects of very low levels of aminoguanidine in protecting cells from the stresses applied in the present study and merits further investigation.

In summary, it is clear that a better understanding of the complex effects of high glucose and aminoguanidine (and similar compounds) on cells is needed. Specifically, a better understanding of membrane interactions, signaling processes, the degree of internalization of aminoguanidine and its intracellular actions is needed. This knowledge will facilitate the effective development of preventive measures for diabetic complications. The present study suggests that elevated D-glucose has deleterious effects both outside and inside retinal capillary cells and that remarkably low, nanomolar, levels of aminoguanidine have favorable effects on retinal capillary cell survival during exposure to high glucose, at least in culture. If the latter findings are borne out in vivo, aminoguandine or similar drugs may have a more favorable "therapeutic index" (relation of safety to efficacy) than has formerly been realized.

\section{ACKNOWLEDGMENT}

This study was supported by grants from the Juvenile Diabetes Foundation, the American Diabetes Association (Lions Clubs Affiliates Sightfirst Program), NIH Research Grant EY-10697 and grants from Alteon Inc. (Ramsey, New Jersey) and the Diabetes Research and Wellness Foundation, Inc. (Fairfax, Virginia).

\section{REFERENCES}

1. Engerman, R.L., 1989. Pathogenesis of diabetic retinopathy. Diabetes, 38: 1203-1206.

2. Merimee, T.J., 1990. Diabetic retinopathy: A synthesis of perspectives. N. Eng. J. Med., 322: 978-983.

3. West, K.M., L.J. Erdreich and J.A. Stober, 1980. A detailed study of risk factors for retinopathy and nephropathy in diabetes. Diabetes, 29: 501-508.

4. Dornan, T.L., A. Ting and C.K. McPherson et al., 1982. Genetic susceptibility to the development of retinopathy in insulin-dependent diabetics. Diabetes, 31: 226-231.

5. Klein, R., B.E. Klein and S.E. Moss et al., 1984. The Wisconsin epidemiologic study of diabetic retinopathy. II. Prevalence and risk of diabetic retinopathy when age at diagnosis is less than 30 years. Arch. Ophthalmol.,102: 520-526.

6. Nathan, D.M., D.E. Singer and J.E. Godine et al., 1986. Retinopathy in older type II diabetics: Association with glucose control. Diabetes, 35: 797-801.

7. The Diabetes Control and Complications Trial Research Group, 1993. The effect of intensive treatment of diabetes on the development and progression of long-term complications in insulindependent diabetes mellitus. N. Engl. J. Med., 329: 977-986. 
8. Lloyd, C.E., R. Klein and R.E. Maser et al., 1995. The progression of retinopathy over 2 years: The Pittsburgh Epidemiology of Diabetes Complications (EDC) study. J. Diab. Complicat., 9: 140-148.

9. Shepro, D. and N.M. Morel, 1993. Pericyte physiology. FASEB J., 7: 1031-1038.

10. Hammes, H.P., 2005. Pericytes and the pathogenesis of diabetic retinopathy. Horm. Metab. Res., 37: 39-43.

11. Thornalley, P.J., M. Westwood and T.W. Lo et al., 1995. Formation of methylglyoxal-modified proteins in vitro and in vivo and their involvement in AGErelated processes. Contrib. Nephrol., 112: 24-31.

12. Lyons, T.J. and A.J. Jenkins, 1997. Glycation, oxidation and lipoxidation in the development of the complications of diabetes mellitus: A carbonyl stress hypothesis. Diab. Rev., 5: 365-391.

13. Requena, J.R., M.U. Ahmed and C.W. Fountain et al., 1997. N-(carboxymethyl) ethanolamine: A biomarker of phospholipid modification by the Maillard Reaction in vivo. J. Biol. Chem., 272: 17473-17479.

14. Papoulis, A., Y. al-Abed and R. Bucala et al., 1995. Identification of N2-(1-carboxyethyl)guanine (CEG) as a guanine advanced glycosylation end product. Biochemistry, 34: 648-655.

15. Hammes, H.P., S. Martin, K. Federlin et al., 1991. Aminoguanidine treatment inhibits the development of experimental diabetic retinopathy. Proc. Natl. Acad. Sci. USA, 88: 11555-11558.

16. Hammes, H.P., D. Strodter and A. Weiss et al., 1995. Secondary intervention with aminoguanidine retards the progression of diabetic retinopathy in the rat model. Diabetologia, 38: 656-660.

17. Agardh, E., B. Hultberg and C. Agardh, 2000. Effects of inhibition of glycation and oxidative stress on the development of cataract and retinal vessel abnormalities in diabetic rats. Curr. Eye Res., 21:543-549.

18. Kern, T.S., J. Tang and M. Mizutani et al., 2000. Response of capillary cell death to aminoguanidine predicts the development of retinopathy: Comparison of diabetes and galactosemia. Invest. Ophthalmol. Vis. Sci., 41: 3972-3978.

19. Kern, T.S. and R.L. Engerman, 2001. Pharmacological inhibition of diabetic retinopathy: Aminoguanidine and aspirin. Diabetes, 50: 1636-1642.

20. Gillies, M.C., T. Su and J. Stayt et al., 1997. Effect of high glucose on permeability of retinal capillary endothelium in vitro. Invest. Ophthalmol. Vis. Sci., 38: 635-642.
21. Panagiotopoulos, S., R.C. O'Brien and R. Bucala et al., 1998. Aminoguanidine has an antiatherogenic effect in the cholesterol-fed rabbit. Atheroscler, 136: 125-131.

22. Edelstein, D. and M. Brownlee, 1992. Rapid Communication: Aminoguanidine ameliorates albuminuria in diabetic hypertensive rats. Diabetologia, 35: 96-97.

23. Yagihashi, S., M. Kamijo and M. Baba et al., 1992. Effect of aminoguanidine on functional and structural abnormalities in peripheral nerve of STZ-induced diabetic rats. Diabetes, 41: 47-52.

24. Zhang, J., S. Ren and D. Sun et al., 1998. Influence of glycation on LDL-induced generation of fibrinolytic regulators in vascular endothelial cells. Arterioscler. Thromb. Vasc. Biol., 18: 1140-1148.

25. Giardino, I., A.K. Fard and D.L. Hatchell et al., 1998. Aminoguanidine inhibits reactive oxygen species formation, lipid peroxidation and oxidantinduced apoptosis. Diabetes, 47: 1114-1120.

26. Corbett, J.A., R.G. Tiltona and K. Chang et al., 1992. Aminoguanidine, a novel inhibitor of nitric oxide formation, prevents diabetic vascular dysfunction. Diabetes, 41: 552-556.

27. Tilton, R.G., K. Chang and K.S. Hasan et al., 1993. Prevention of diabetic vascular dysfunction by guanidines: Inhibition of nitric oxide synthase versus advanced glycation end-product formation. Diabetes, 42: 221-232.

28. Ivanova, S., G.I. Botchkina and Y. Al-Abed et al., 1998. Cerebral ischemia enhances polyamine oxidation: Identification of enzymatically formed 3-aminopropanal as an endogenous mediator of neuronal and glial cell death. J. Exp. Med., 188: 327-340.

29. Chakravarthy, U., A.W. Stitt and J. McNally et al., 1995. Nitric oxide synthase activity and expression in retinal capillary endothelial cells and pericytes. Curr. Eye Res., 14: 285-294.

30. Hoffmann, G., S. Kenn and B. Wirleitner et al., 1998. Neopterin induces nitric oxide-dependent apoptosis in rat vascular smooth muscle cells. Immunobiology, 199:63-73.

31. Cagliero, E., T. Roth and S. Roy et al., 1991. Characteristics and mechanisms of high-glucoseinduced overexpression of basement membrane components in cultured human endothelial cells. Diabetes, 40: 102-110.

32. Bucala, R., Z. Makita and G. Vega et al., 1994. Modification of low density lipoprotein by advanced glycation end products contributes to the dyslipidemia of diabetes and renal insufficiency. Proc. Natl. Acad. Sci. USA, 91: 9441-9445. 
33. Lyons, T.J., W. Li and M.C. Wells-Knecht et al., 1994. Toxicity of mildly modified low density lipoproteins to cultured retinal capillary endothelial cells and pericytes. Diabetes, 43: 1090-1095.

34. King, G.I., S.M. Buzney and R. Kahn et al., 1983. Differential responsiveness to insulin of endothelial and support cells from micro- and macrovessels. J. Clin. Invest., 71: 974-979.

35. Nayak, R.C., A.B. Berman and K.L. George et al., 1988. A monoclonal antibody (3G5)-defined ganglioside antigen is expressed on the cell surface of microvascular pericytes. J. Exp. Med., 167: 1003-1015.

36. Markwell, M.A., L.L. Biever and N.E. Tolbert, 1978. A modification of the Lowry procedure to simplify protein determination in membrane and lipoprotein samples. Anal. Biochem., 87: 206-211.

37. Chibber, R., P.A. Molinatti and J.S.K. Wong et al., The effect of aminoguanidine and tolrestat on glucose toxicity in bovine retinal capillary pericytes. Diabetes, 43: 758-763.

38. Malek, A.M., G.G. Goss and L. Jiang et al., 1998. Mannitol at clinical concentrations activates multiple signaling pathways and induces apoptosis in endothelial cells. Stroke, 29: 2631-2640.

39. Baynes, J.W., 1991. Role of oxidative stress in development of complications in diabetes. Diabetes, 40: 405-412.

40. Qin, S., Y. Minami and M. Hibi et al., 1997. Sykdependent and -independent signaling cascades in B cells elicited by osmotic and oxidative stress. J. Biol. Chem., 272: 2098-2103.

41. Brownlee, M., 2001. Biochemistry and molecular cell biology of diabetic complications. Nature, 414: 813-820.

42. Amano, S., S. Yamagishi and Y. Inagaki et al., Pigment epithelium-derived factor inhibits oxidative stress-induced apoptosis and dysfunction of cultured retinal pericytes. Microvasc. Res., 69: 45-55.

43. Picard, S., S. Parthasarathy and J. Freubis et al., 1992. Aminoguanidine inhibits oxidative modification of low density lipoprotein protein and the subsequent increase in uptake by macrophage scavenger receptors. Proc. Natl. Acad. Sci. USA, 88: 6876-6880.
44. Philis-Tsimikas, A., S. Parthasarathy and S. Picard et al., 1995. Aminoguandine has both prooxidant and antioxidant activity toward LDL. Arterioscler. Thromb. Vasc. Biol., 15: 367-376.

45. Koya, D. and G.L. King, 1998. Protein kinase C activation and the development of diabetic complications. Diabetes, 47: 859-866.

46. Knecht, K.J., M.S. Feather and J.W. Baynes et al., 1992. Detection of 3-deoxyfructose and 3deoxyglucosone in human urine and plasma: Evidence for intermediate stages of the Maillard reaction in vivo. Arch. Biochem. Biophys., 294: 130-137.

47. Kim, J., J.W. Son and J.A. Lee et al., 2004. Methylglyoxal induces apoptosis mediated by reactive oxygen species in bovine retinal pericytes. J. Korean Med. Sci., 19: 95-100.

48. Liu, B., M. Bhat and A.K. Padival et al., 2004. Effect of dicarbonyl modification of fibronectin on retinal capillary pericytes. Invest. Ophthalmol. Vis. Sci., 45: 1983-1995.

49. Du, Y., M.A. Smith and C.M. Miller et al., 2002. Diabetes-induced nitrative stress in the retina and correction by aminoguanidine. J. Neurochem., 80: 771-779.

50. Miller, A.G., D.G. Smith and M. Bhat et al., 2006. Glyoxalase I is critical for human retinal capillary pericyte survival under hyperglycemic conditions. J. Biol. Chem., 281: 11864-11871.

51. Yu, P.H. and D.M. Zuo, 1997. Aminoguanidine inhibits semicarbazide-sensitive amine oxidase activity: Implications for advanced glycation and diabetic complications. Diabetologia, 40: 1243-1250.

52. Lyons, T.J., W. Li and B. Wojciechowski et al., 2000. Aminoguanidine and the effects of modified LDL on cultured retinal capillary cells. Invest. Ophthalmol. Vis. Sci., 41: 1176-1180.

53. Roth, M.G. and P.C. Sternweis, 1997. The role of lipid signaling in constitutive membrane traffic. Curr. Opin. Cell Biol., 9: 519-526. 